\title{
Evolução da mortalidade neonatal no Estado do Rio de Janeiro, Brasil, de 1979 a 1993.1 - Análise por grupo etário segundo região de residência
}

\author{
Progress of neonatal mortality in a metropolitan area of \\ Southeastern Brazil from 1979 to 1993. \\ 1 - Analysis by age-group according to area of residence
}

\section{Maria do Carmo Leal e Célia Landman Szwarcwald}

Escola Nacional de Saúde Pública. Rio de Janeiro, RJ - Brasil (M.C.L.), Departamento de Informações para a Saúde da Fundação Oswaldo Cruz. Rio de Janeiro, RJ - Brasil (C.L.S.)

\begin{abstract}
Resumo
A análise do comportamento da mortalidade infantil no Estado do Rio de Janeiro, no período de 1979 a 1993, mostra evolução de decréscimo, com ritmo bem mais lento de declive no componente neonatal do que no componente tardio. $\mathrm{O}$ coeficiente de mortalidade neonatal apresenta ainda menor velocidade de queda ao se subtrair do numerador os óbitos ocorridos por desnutrição, doenças diarréicas e pneumonias. Examinando-se os dados por região de residência, é o Interior do Estado que apresenta o pior desempenho. Categorizando-se por idade da criança, observa-se um padrão no qual, dentro de cada grupo de idade, é sempre na faixa etária inferior que o declínio é menos acentuado. Em contraste ao que ocorre nos países mais industrializados, a mortalidade infantil no Brasil decresce tanto menos quanto mais se aproxima do momento do nascimento, observando-se, inclusive, tendências de aumento para o grupo de óbitos ocorridos até uma hora após o parto em todas as três regiões de residência consideradas no estudo. Para o enfrentamento desta situação se faz necessária uma reestruturação dos serviços de saúde, tanto na melhoria da qualidade da assistência à gestação e ao parto, quanto no desenvolvimento de estratégias de monitoramento epidemiológico da realidade do País.
\end{abstract}

Mortalidade infantil, tendências. Mortalidade neonatal. Qualidade dos cuidados de saúde.

\begin{abstract}
The behavior of the infant mortality rate in the State of Rio de Janeiro, Brazil, from 1979 to 1993, is analysed. The annual rate of variation, calculated as the slope of an exponential regression model, indicates an overall decreasing trend of approximately 5\% per year. Although the neonatal component is also decreasing, the annual rate of variation is much lower, of only $2,4 \%$ per year, and is even lower (1,6\%) when deaths caused by malnutrition or infectious
\end{abstract}

Correspondência para/Correspondence to: Maria do Carmo Leal - Escola Nacional de Saúde Pública. Av. Leopoldo Bulhões, 1480 - Sala 818 -

21041-210 Rio de Janeiro, RJ - Brasil. Fax: (021) 280.8194 E-mail: docarmo@dcc001,cict.fiocruz.br

Recebido em 29.9.1995. Reapresentado em 23.1.1996. Aprovado em 28.2.1996. 
diseases are not taken into account. Examining date by region of residence, the area composed of the counties located outside the metropolitan region-called the "Interior" - presents the worst perfomance. When mortality rates are analysed by age-group an interesting pattern is found: the shorter the time of life the lower the value of the rate variation. In contrast to what is found in developed countries, where care is more intensive for newborn children, the infant mortality rate in Rio de Janeiro State dedreases less as the number of hours of life approaches the delivery: for the groups of deathas that occurred within the first of life, an increasing trend is found in all of the residencial regions considered in this study. To face this situation, it is necessary to reaffirm the priorities of the health services, not only in respect to the improvement of the quality of medical assistance but also to the development of strategies for the epidemiological monitoring of the Brazilian situation

Infant mortality, trends. Neonatal mortality. Quality of health care.

\section{INTRODUÇÃO}

O coeficiente de mortalidade infantil é um indicador de saúde que, além de informar sobre os níveis de saúde de uma população, sintetiza as condições de bem-estar social, político e ético de uma dada conformação social. Isto porque ele significa a probabilidade de sobrevivência infantil no primeiro ano de vida e por isto mesmo reflete não só as condições concretas de moradia, salário, alimentação, atenção à saúde, mas também, e talvez, principalmente o compromisso de uma dada sociedade com a sua reprodução social. Ou seja, em que medida a sociedade protege a sua renovação geracional ${ }^{21}$.

Certamente esta interpretação sobre o coeficiente de mortalidade infantil não se aplica a toda à sua evolução histórica. Mas contextualizada nos dias de hoje, em que a mortalidade infantil é vista, em princípio, como um evento evitável e traçador da qualidade dos serviços de saúde, mostra-se absolutamente pertinente e adequada. O conceito de evitabilidade de um problema de saúde foi desenvolvido por Rutstein e col. ${ }^{18}$, nos Estados Unidos, nas décadas de 30 a 60, inspirado na experiência da redução da mortalidade infantil naquele país, a partir da intervenção dos serviços de saúde.

Mesmo nos países industrializados, nos quais a mortalidade infantil já atingiu níveis muito baixos, este é ainda considerado problema prioritário de saúde pública devido a evitabilidade da sua ocorrência ser, em grande parte, definida comprovadamente pela qualidade da atenção médica e das intervenções sanitárias ${ }^{12}$. Passível, portanto, de uma atuação setorial específica da saúde.
Muito embora não se possa nem se queira ignorar as determinações sociais mais amplas que definem riscos de morrer diferenciados, no Brasil, as enormes diferenças verificadas na mortalidade infantil por grupos sociais ${ }^{13,17}$ reproduzem-se e se amplificam porque o acesso aos serviços de saúde têm a mesma desigualdade que caracteriza a nossa sociedade. Ao contrário do desejável, os serviços de saúde distinguem-se por uma seletividade social tanto no acesso, quanto na qualidade e resolutividade das suas intervenções. Assim sendo, a eficácia e efetividade das suas práticas não têm podido se estender aos grupos que mais necessitam da sua ação promotora, protetora e reabilitadora da saúde ${ }^{19}$.

A diminuição nos coeficientes de mortalidade infantil que vem se verificando nos últimos 15 anos, período para o qual se dispõe de dados nacionais, não tem atenuado os diferenciais por grupos sociais que são da ordem de 5 a 6 vezes entre o maior e o menor grupo de renda ${ }^{17,23}$. Esta queda deu-se principalmente sobre o componente pós-neonatal da mortalidade infantil, como mostrado por Szwarcwald ${ }^{27} \mathrm{e}$ Duchiade $^{5}$. Dentre as causas, a diarréia foi a que mais decresceu. Observou-se também, nesse período, tendência à constância nas taxas da mortalidade neonatal.

O presente trabalho tomou como objeto de estudo a tendência temporal da mortalidade neonatal no Estado do Rio de Janeiro para o período de 1979 a 1993, comparando-a com a evolução da mortalidade pós-neonatal. O confronto com a experiência de outros países e do acompanhamento dos indicadores sociais e de assistência médica no, mesmo período, buscou identificar possíveis explicações para o seu comportamento. 


\section{METODOLOGIA}

No presente estudo, para comparação da evolução da mortalidade infantil por região de residência da criança, o Estado do Rio de Janeiro foi subdividido em três grandes áreas: o Município da Capital, o Cinturão Metropolitano do Rio de Janeiro - constituído pelos municípios de Duque de Caxias, Itaboraí, Itaguaí, Magé, Mangaratiba, Maricá, Nilópolis, Niterói, Nova Iguaçu, Paracambi, São Gonçalo, São João do Meriti - e o Interior, composto pelos demais municípios do Estado. No decorrer da década de 80, a Fundação Instituto Brasileiro de Geografia e Estatística (FIBGE) alterou a composição dos dois últimos estratos - o Cinturão Metropolitano e o Interior - por isto, esclarece-se que se optou por manter para todo o período do estudo o critério inicial de definição dessas áreas, utilizado por aquele órgão, de forma a viabilizar uma comparação temporal.

Como é amplamente conhecido, o coeficiente de mortalidade infantil é dado pelo número de óbitos em menores de um ano de idade em um determinado ano e lugar para cada mil nascidos vivos naquela mesma área e período. Os óbitos ocorridos nos menores de 28 dias de vida constituem o componente neonatal da mortalidade infantil, os ocorridos do $28^{\circ}$ dia em diante compõem a mortalidade pós-neonatal.

Para se efetivar o cálculo correto da mortaidade infantil é necessário que se disponha de informações confiáveis para compor o numerador e denominador desta taxa. Em relação ao número de óbitos em menores de um ano, utilizou-se como fonte de informações o Subsistema de Informações sobre Mortalidade do Ministério da Saúde (SIM/ MS), para o período de 1979 a 1990. Quanto aos anos de 1991 a 1993, os dados foram cedidos pela Secretaria Estadual de Saúde do Rio de Janeiro.

Apesar de não se ter estimativas precisas da cobertura do SIM/MS no Estado do Rio de Janeiro, sabe-se que se trata de um dos mais antigos e bem estruturados do País e que o sub-registro de óbitos é de pequena magnitude. Sendo assim, no presente estudo, o número de óbitos foi considerado igual ao número de óbitos reportados, sem qualquer correção para eventuais subnotificações de mortes entre os menores de um ano. Foram analisados os óbitos ocorridos nos residentes em municípios pertencentes ao Estado do Rio de Janeiro.

Para obter o número de nascidos vivos a cada ano, utilizou-se as informações das Estatísticas do Registro Civil da FIBGE ${ }^{8}$. Diferentemente dos óbitos, a proporção de nascidos vivos não registrados no ano de nascimento é de relevância, tendo sido necessário desenvolver um modelo para corrigir o efeito do registro fora de prazo.

Nas referidas publicações ${ }^{6}$, o número total de registros em um determinado ano é apresentado segundo ano de nascimento, se este tiver ocorrido até 8 anos antes da data da publicação. Sob as suposições de que uma parcela não significativa da população é registrada depois de 8 anos do nascimento e que não há "registro duplo" durante este período, deduz-se que:

$$
\mathrm{N}_{\mathrm{t}}=\mathrm{R}_{\mathrm{t}, \mathrm{t}}+\mathrm{R}_{\mathrm{t}, \mathrm{t}+1}+\ldots \ldots+\mathrm{R}_{\mathrm{t}, \mathrm{t}+8}
$$

sendo $\mathbf{N}_{\mathrm{t}}$ o número de nascidos vivos no ano $\mathbf{t}$ e $\mathbf{R}_{\mathrm{t}, \mathrm{t}+\mathrm{j}}$ é o número de nascidos vivos no ano $\mathbf{t}$ e registrados no ano $(\mathbf{t}$ $+\mathbf{j})$ para $\mathbf{j}=\mathbf{0}, \ldots . . . . . .8 .8$.

Dispondo-se das informações do Registro Civil para o período de 1979 a 1991, através da expressão acima foi possível corrigir o número de nascidos vivos para os anos de 1979 a 1983, para cada uma das três regiões do Estado consideradas no presente estudo. Os fatores de correção no ano $\mathbf{t}$, definidos como $\mathbf{f}_{\mathbf{t}}=\mathbf{N}_{\mathbf{t}} / \mathbf{R}_{\mathrm{t}, \mathrm{t}}$, mantiveram-se aproximadamente invariantes nesses 5 anos em todas as regiões, não apresentando tendências de melhora do registro de nascimento. Sendo assim, para cada região os fatores de correção foram calculados como média aritmética dos fatores $\mathrm{f}_{\mathrm{t}}$ para $\mathbf{t}$ variando de 1979 a 1983 , obtendo-se respectivamente 1.177 para a Capital, 1.284 para o Cinturão Metropolitano e 1.223 para o Interior.

Supondo-se que a subenumeração de nascidos vivos permanece constante também entre 1985 e 1991, utilizaram-se os mesmos fatores de correção para obter estimativas corrigidas do número de nascidos vivos, em cada região, no período de 1979 a 1991. Em uma segunda etapa, para corrigir o efeito das flutuações casuais das estatísticas de registro, e também para obter estimativas para os anos de 1992 e 1993, dos quais não se dispunha de informações, utilizou-se um modelo de regressão exponencial em cada uma das três regiões.

Os nascidos vivos corrigidos primeiramente pelo fator médio de correção (obtido no período 1979-83) e posteriormente ajustados pelo modelo de regressão exponencial forneceram, então, as estimativas finais dos denominadores dos coeficientes de mortalidade infantil utilizados no presente trabalho.

Para melhor entendimento, as etapas de procedimento foram exemplificadas, passo a passo, para o Município do Rio de Janeiro, na Tabela 1. O número estimado de nascidos vivos, de 1979 a 1993, nas três regiões, está apresentado na Tabela 2.

A análise estatística dos dados consistiu em avaliar o comportamento temporal da mortalidade neonatal segundo região de residência e a idade da criança, confrontando os resultados com estatísticas de outros países. A evolução da mortalidade pós-neonatal foi utilizada como um parâmetro de comparação.

Para caracterizar cada série temporal, calculou-se a "taxa percentual (\%) de variação anual" definida como o coeficiente angular da regressão exponencial ajustada aos valores observados de 1979 a 1993, multiplicado por 100 . A significância da taxa (\%) de variação anual foi obtida através do teste da hipótese nula de que o coeficiente angular da regressão é zero. Valores foram considerados significativos para $\mathrm{p}<5 \%$.

Sem entrar em detalhamento da análise da mortalidade neonatal por causa de óbito, que será objeto de um outro artigo, considerou-se apenas a contribuição das causas infecciosas e da desnutrição. Para isto excluiu-se da taxa de mortalidade neonatal os óbitos por doenças diarréicas ( CID 001-009), por pneumonias ( CID 480-486 ) e por 
Tabela 1 - Exemplificação do procedimento utilizado para estimar o número de nascidos vivos de 1979 a 1993. Município do Rio de Janeiro.

\begin{tabular}{|c|c|c|c|c|c|}
\hline Ano & $R_{t, t}$ & $\mathrm{~N}_{\mathrm{t}}$ & $f_{t}$ & $R_{t, t} \times f^{(1)}$ & $y_{t}^{(2)}$ \\
\hline 1979 & 100.232 & 119.352 & 1,191 & 117.973 & 122.426 \\
\hline 1980 & 100.872 & 118.252 & 1,172 & 118.726 & 120.307 \\
\hline 1981 & 105.409 & 123.584 & 1,172 & 124.066 & 118.224 \\
\hline 1982 & 103.989 & 121.543 & 1,169 & 122.395 & 116.178 \\
\hline 1983 & 97.241 & 114.681 & 1,179 & 114.453 & 114.167 \\
\hline 1984 & 93.575 & - & - & 110.138 & 112.191 \\
\hline 1985 & 92.171 & - & - & 108.485 & 110.249 \\
\hline 1986 & 91.652 & - & - & 107.874 & 108.341 \\
\hline 1987 & 85.794 & - & - & 100.980 & 106.466 \\
\hline 1988 & 90.161 & - & - & 106.119 & 104.623 \\
\hline 1989 & 90.767 & - & - & 106.833 & 102.812 \\
\hline 1990 & 83.801 & - & - & 98.634 & 101.033 \\
\hline 1991 & 84.032 & - & - & 98.906 & 99.284 \\
\hline 1992 & - & - & - & - & 97.565 \\
\hline 1993 & - & - & - & - & 95.877 \\
\hline
\end{tabular}

(1) $f=1 / 5 \cdot \sum f_{t}=1,177$

(2) $=$ Valores ajustados pela regressão exponencia

Fontes: Sistema de Informações sobre Mortalidade do Ministério da Saúde (1979-1990) - Secretaria Estadual de Saúde do Rio de Janeiro (1991-1993).

Tabela 2 - Número estimado de nascidos vivos por região de residência. Estado do Rio de Janeiro, 1979-1993.

\begin{tabular}{ccccc}
\hline Anos & Município do Rio & Cinturão Metropolitano & Interior & Estado \\
\hline 1979 & 122.426 & 104.823 & 73.862 & 301.111 \\
1980 & 120.307 & 103.989 & 72.572 & 296.868 \\
1981 & 118.224 & 103.162 & 71.303 & 292.689 \\
1982 & 116.178 & 102.341 & 70.057 & 288.576 \\
1983 & 114.167 & 101.526 & 68.833 & 284.526 \\
1984 & 112.191 & 100.718 & 67.630 & 280.539 \\
1985 & 110.249 & 99.917 & 66.448 & 276.614 \\
1986 & 108.341 & 99.122 & 65.287 & 272.750 \\
1987 & 106.466 & 98.333 & 64.146 & 268.945 \\
1988 & 104.623 & 97.550 & 63.025 & 265.198 \\
1989 & 102.812 & 96.774 & 61.924 & 261.510 \\
1990 & 101.033 & 96.004 & 60.842 & 257.879 \\
1991 & 99.284 & 95.240 & 59.778 & 254.302 \\
1992 & 97.565 & 94.482 & 58.734 & 250.781 \\
1993 & 95.877 & 93.730 & 57.707 & 247.314 \\
\hline
\end{tabular}

Fontes: Sistema de Informações sobre Mortalidade do Ministério da Saúde (1979-1990) - Secretaria Estadual de Saúde do Rio de Janeiro (1991-1993).

desnutrição protéico-calórica (CID 260-263) e analisouse o comportamento da "mortalidade neonatal excluída" diante dos resultados obtidos previamente.

\section{RESULTADOS}

No período estudado, o coeficiente de mortalidade infantil (CMI) decresceu de cerca de 5\% ao ano no Estado do Rio de Janeiro, apresentando em 1979 taxa de 50,8, por 1.000 nascidos vivos (NV), e em 1993, de 29,0 (Tabela 3). Observa-se que o decréscimo não foi homogêneo nas diversas regiões. Assim, no Cinturão Metropolitano, que tinha ao início do período as taxas mais elevadas, de 64,5 por 1.000 $\mathrm{NV}$, o decréscimo foi de $6 \%$ ao ano, chegando a alcançar, em 1993, o valor de 26,7. Este último coefi- ciente é muito semelhante ao que se observa no Município da Capital que tem para o mesmo ano taxa de 23,3. Esses dados merecem destaque por que no ano de 1979, ano inicial da série histórica, o Município da Capital já se encontrava em uma situação privilegiada em relação ao Cinturão Metropolitano, com taxa de 37,4 por $1.000 \mathrm{NV}$, quase a metade do observado naquela área. No entanto, a situação mais alarmante é encontrada, sem dúvida, no Interior do Estado que, ao início do período, apresentava um coeficiente mais baixo que o Cinturão Metropolitano, de 53,6 por $1.000 \mathrm{NV}$ e, ao final, mostrou-se numa situação muito mais desvantajosa, com valores de 42,0 por $1.000 \mathrm{NV}$, exibindo uma taxa de decréscimo de apenas 3,6\% ao ano, a menor do Estado. 
Tabela 3 - Coeficiente de mortalidade infantil (óbitos \%o nv) segundo região de residência. Rio de Janeiro, 1979-1993.

\begin{tabular}{|c|c|c|c|c|c|c|c|c|c|c|c|c|c|c|}
\hline Regiões & 1979 & 1980 & 1981 & 1982 & 1983 & 1984 & 1985 & 1986 & 1987 & 1988 & 1989 & 1990 & 1991 & 19921993 \\
\hline Município do Rio & 37,4 & 36,1 & 35,4 & 32,1 & 29,2 & 29,2 & 26,0 & 27,3 & 23,5 & 27,4 & 22,7 & 22,9 & 19,7 & $21,0 \quad 23,3$ \\
\hline Cint. Metropolitano & 64,5 & 58,5 & 59,8 & 53,4 & 47,5 & 52,4 & 42,7 & 42,1 & 37,0 & 43,2 & 37,0 & 35,1 & 28,4 & $31,2 \quad 26,7$ \\
\hline Interior & 53,6 & 58,4 & 56,0 & 52,5 & 52,0 & 47,2 & 42,7 & 42,0 & 39,6 & 40,0 & 37,1 & 43,5 & 31,2 & $33,3 \quad 42,0$ \\
\hline Estado & 50,8 & 49,4 & 49,0 & 44,6 & 41,4 & 41,9 & 36,0 & 36,2 & 32,3 & 36,2 & 31,4 & 32,3 & 25,7 & $27,7 \quad 29,0$ \\
\hline
\end{tabular}

Fontes: Sistema de Informações sobre Mortalidade do Ministério da Saúde (1979-1990) - Secretaria Estadual de Saúde do Rio de Janeiro (1991-1993).

Tabela 4 - Taxa (\%) de variação anual medida dos coeficientes de mortalidade neonatal e pós-neonatal. Rio de Janeiro, 1979-1993.

\begin{tabular}{lccc}
\hline Regiões & Neonatal & Pós-neonatal & Total \\
\hline Município do Rio & $-2,59$ & $-6,35$ & $-4,22$ \\
Cint. Metropolitano & $-3,10$ & $-8,77$ & $-5,94$ \\
Interior & $-1,43$ & $-6,19$ & $-3,63$ \\
\hline Estado & $-2,41$ & $-7,28$ & $-4,69$ \\
\hline
\end{tabular}

Fontes: Sistema de Informações sobre Mortalidade do Ministério da Saúde (1979-1990) - Secretaria Estadual de Saúde do Rio de Janeiro (1991-1993).

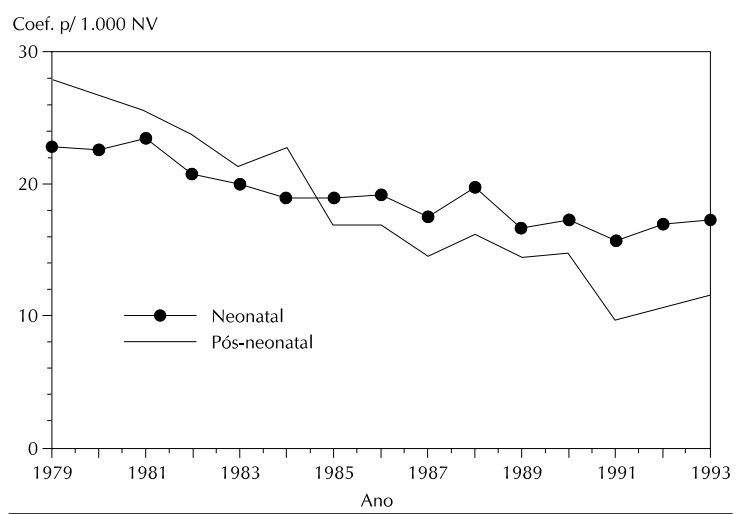

Figura 1 - Coeficientes de mortalidade neonatal e pósneonatal. Estado do Rio de Janeiro, 1979-1993.

Prioritariamente, a queda do CMI no Estado do Rio de Janeiro fez-se às custas do componente pósneonatal. Para os maiores de 27 dias, o decréscimo foi de 7,3\% ao ano, enquanto para os óbitos neonatais o decréscimo foi apenas de $2,4 \%$ (Tabela 4). Comparando a evolução por região, o Cinturão Metropolitano foi o que apresentou a maior queda do componente pós-neonatal, de 8,8\% ao ano, seguido do $\mathrm{Mu}$ nicípio da Capital e do Interior que tiveram taxas de declínio semelhantes de 6,4 e 6,2\%, respectivamente. O impacto quantitativo que estes decréscimos fazem nas taxas de mortalidade infantil, observadas segundo seus componentes, são realmente impressionantes. No Estado, o coeficiente de mortalidade pósneonatal reduziu-se de 28,0 por $1.000 \mathrm{NV}$ para 11,6 , passando a ser menor do que o coeficiente de mortalidade neonatal no final do período (Figura 1).

Da mesma forma que para a mortalidade pósneonatal, de todas as áreas analisadas, foi o Cinturão

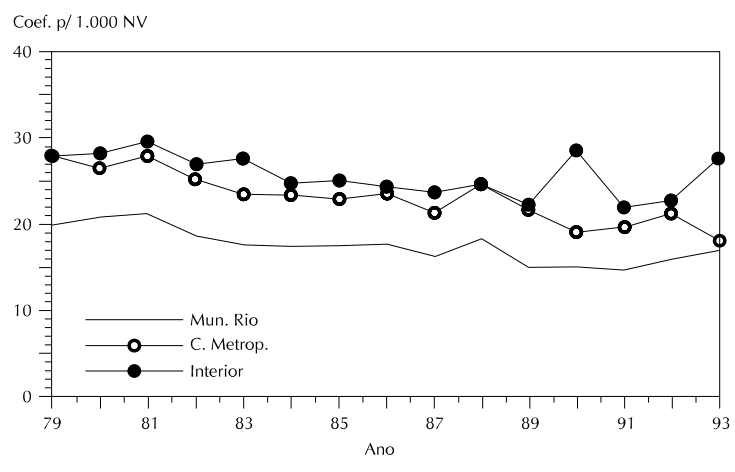

Figura 2 - Coeficientes de mortalidade neonatal por região de residência. Estado do Rio de Janeiro, 1979-1993.

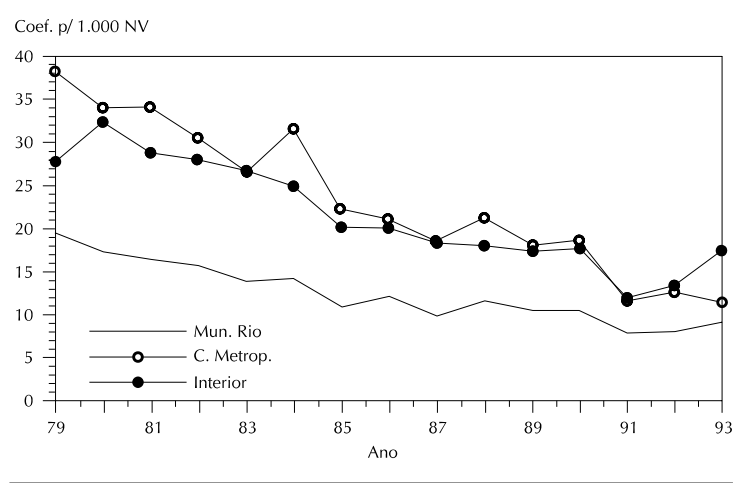

Figura 3 - Coeficientes de mortalidade pós-neonatal por região de residência. Estado do Rio de Janeiro, 1979-1993.

Metropolitano a que mostrou maior tendência de queda na mortalidade neonatal, de $3,1 \%$ ao ano, evoluindo de uma taxa de 26,3 em 1979 para 15,6 por 1.000 NV em 1993 (Figuras 2, 3). No final do período, esses valores se aproximaram dos encontrados na Capital que, apresentando decréscimo anual menor, de 2,6\%, decaiu bem menos, de 17,9, em 1979, para $14,5 \mathrm{em}$ 1993. Chama a atenção o que vem ocorrendo no Interior do Estado, já que é exatamente no componente neonatal que o desempenho da mortalidade infantil é mais precário. O decréscimo verificado é muito pequeno, de apenas $1,4 \%$ ao ano, menos da metade do ocorrido no Cinturão Metropolitano e bem aquém do Município da Capital. A série temporal no Interior tem início em 25,9 e termina em 25,0 por $1.000 \mathrm{NV}$, permanecendo praticamente constante. 
Tabela 5 - Mortalidade proporcional (\%) nos menores de um ano de idade por horas e dias de vida. Estado do Rio de Janeiro, 1979-1993.

\begin{tabular}{|c|c|c|c|c|c|c|c|c|c|c|c|c|c|c|}
\hline Idade & 1979 & 1980 & 1981 & 1982 & 1983 & 1984 & 1985 & 1986 & 1987 & 1988 & 1989 & 1990 & 1991 & 19921993 \\
\hline$<1$ hora & 4,8 & 4,9 & 5,2 & 4,8 & 5,7 & 4,5 & 4,6 & 6,3 & 6,4 & 6,0 & 7,2 & 9,1 & 11,3 & $11,5 \quad 9,2$ \\
\hline $1-6$ ho & 4,0 & 4,4 & 4,9 & 4,6 & 4,7 & 5,1 & 6,3 & 6,6 & 6,9 & 6,9 & 6,8 & 6,5 & 6,5 & 7,0 \\
\hline 7-23 hor & 6,0 & 6,2 & 6,9 & 6,5 & 6,5 & 6,0 & 6,9 & 6,6 & 7,0 & 7,2 & 8,4 & 7,5 & 9,1 & 8,4 \\
\hline$<1$ dia & 14,8 & 15,5 & 17,0 & 15,9 & 16,9 & 15,6 & 17,8 & 19,5 & 20,3 & 20,1 & 22,4 & 23,1 & 26,9 & $27,2 \quad 24,6$ \\
\hline 1-6 dias & 16,8 & 18,2 & 19,5 & 19,2 & 20,3 & 18,9 & 22,8 & 21,1 & 22,0 & 21,4 & 19,7 & 20,2 & 23,7 & $23,9 \quad 24,1$ \\
\hline $7-27$ dias & 13,2 & 12,1 & 11,4 & 11,4 & 11,0 & 11,1 & 12,2 & 12,3 & 12,2 & 13,4 & 11,4 & 10,6 & 11,4 & $10,7 \quad 11,2$ \\
\hline 28-364 dias & 55,2 & 54,2 & 52,1 & 53,5 & 51,8 & 54,4 & 47,2 & 47,1 & 45,5 & 45,1 & 46,5 & 46,1 & 38,0 & $38,2 \quad 40,1$ \\
\hline
\end{tabular}

Fontes: Sistema de Informações sobre Mortalidade do Ministério da Saúde (1979-1990) - Secretaria Estadual de Saúde do Rio de Janeiro (1991-1993).

Ao se analisar a mortalidade infantil por dias e semanas após o nascimento, observa-se que ocorreu aumento na proporção dos óbitos que aconteceram no primeiro dia de vida (Tabela 5). No ano de 1979, representavam $14,8 \%$ do total dos óbitos ocorridos no Estado e em 1993 já somavam 24,6\%. No grupo de 1-6 dias também verifica-se uma tendência semelhante de aumento, enquanto nos outros dois estratos, de 7-27 dias e 28dias e mais, há diminuição.

Assim, a evolução temporal do CMI por faixa etária, apresenta declives significativos $(p<0,01)$ nos grupos etários de 7-27 dias e de de 28 dias e mais, mas não para os menores de um dia. Este padrão repete-se para o Cinturão Metropolitano e o Interior do Estado. A única exceção é o Município do Rio de Janeiro que apresenta decréscimos significativos para as três faixas de idade sob consideração (Tabela 6).

Tendo em vista o comportamento atípico dos menores de um dia, para melhor analisá-los, foram subdivididos em três grupos: os que morreram com menos de uma hora de vida, entre 1 e 6 horas e entre 6 e 23 horas. Através dos dados dispostos na Tabela 5 verifica-se que houve aumento nas proporções de óbitos ocorridos nos dois primeiros grupos etários. Analisando a evolução do CMI por horas vividas, para a totalidade do Estado (Tabela 6), observa-se uma diminuição significativa, de $2,2 \%$ ao ano, para

Tabela 6 - Taxa (\%) de variação anual dos coeficientes de mortalidade infantil por dias e horas vividas segundo região de residência. Estado do Rio de Janeiro, 1979-1993.

\begin{tabular}{lcccc}
\hline $\begin{array}{l}\text { Horas de } \\
\text { vida }\end{array}$ & $\begin{array}{c}\text { Município } \\
\text { do Rio }\end{array}$ & $\begin{array}{c}\text { Cinturão } \\
\text { Metropolitano }\end{array}$ & Interior & Estado \\
\hline$<1$ hora & $0,97^{*}$ & 0,94 & $2,95^{*}$ & $1,70^{*}$ \\
1-6 horas & $-2,14$ & $-0,18^{*}$ & $0,49^{*}$ & $-0,52^{*}$ \\
$6-23$ horas & $-2,71$ & $-2,47$ & $-0,79^{*}$ & $-2,18$ \\
\hline$<1$ dia & $-1,39$ & $-0,88^{*}$ & $-0,60^{*}$ & $-0,38^{*}$ \\
\hline 1-6 dias & $-3,28$ & $-3,23$ & $-1,81$ & $-2,78$ \\
7-27 dias & $-3,72$ & $-7,10$ & $-4,63$ & $-5,30$ \\
28 dias e mais & $-6,35$ & $-8,77$ & $-6,19$ & $-7,28$ \\
\hline
\end{tabular}

* Não significativo ao nível de $5 \%$.

Fontes: Sistema de Informações sobre Mortalidade do Ministério da Saúde (19791990) - Secretaria Estadual de Saúde do Rio de Janeiro (1991-1993). as taxas relativas aos óbitos ocorridos entre 6 e 23 horas, uma estabilidade para o grupo de 1 a 6 horas, e um aumento de $1,7 \%$ ao ano para o de menos de uma hora de vida. No Município do Rio de Janeiro, verifica-se discreto aumento nesta última faixa de idade (menos de uma hora). O Cinturão Metropolitano apresenta queda significativa apenas para o grupo de 6 a 23 horas. De todas as áreas é o Interior do Estado que apresenta a pior situação, pois não teve nenhuma diminuição que se mostrasse significativa em nenhuma das faixas de idade analisadas, ao contrário, mostrou um crescimento significativo de $3 \%$ ao ano no grupo de óbitos ocorrido até uma hora após o nascimento.

A exclusão das doenças infecciosas e da desnutrição do coeficiente de mortalidade neonatal tem o significado de retirar dele o que é característico da mortalidade pós-neonatal. Quando se exclui estas causas (conforme descrito na metodologia), observase uma redução muito grande na intensidade da queda do coeficiente de mortalidade neonatal no período de 1979 a 1993 (Tabela 7). O Estado do Rio de Janeiro, que apresentava declive de $2,4 \%$ para o período neonatal como um todo, com a exclusão passa a mostrar uma taxa de descenso de apenas $1,6 \%$. Padrões similares são observados para todas as regiões de residência. A diferença entre as três áreas é que se na Capital a tendência de queda se modifica pouco, no Interior, se reduz muito, tornando-se, inclusive, não significativa.

Tabela 7 - Taxa (\%) de variação anual dos coeficientes de mortalidade neonatal e neonatal excluída* por região. Estado do Rio de Janeiro, 1979-1993.

\begin{tabular}{lcc}
\hline Região de residência & Neonatal & Neonatal excluída \\
\hline Município do Rio & $-2,59$ & $-2,03$ \\
Cinturão Metropolitano & $-3,10$ & $-1,96$ \\
Interior & $-1,43$ & $-0,49 * *$ \\
\hline Estado & $-2,41$ & $-1,56$ \\
\hline
\end{tabular}

* Excluídas: diarréias, pneumonias e desnutrição.

** Não significativo ao nível de 5\%.

Fontes: Sistema de Informações sobre Mortalidade do Ministério da Saúde (19791990) - Secretaria Estadual de Saúde do Rio de Janeiro (1991-1993). 
Evidencia-se dos resultados encontrados que, quando se examina os dados por sub-região do Estado, é o Interior que apresenta o pior desempenho no período estudado. O decréscimo da mortalidade infantil foi aquém das outras áreas tanto no período neonatal quanto no pós-neonatal. Já quando se analisa a tendência de queda da mortalidade infantil por faixa etária, verifica-se a ocorrência de um padrão em que, dentro de cada estrato de idade, é sempre na faixa de maior idade que a queda da mortalidade infantil é maior. Assim, entre os menores de um ano, o declínio mais acentuado é observado entre os maiores de 27 dias; entre os menores de um mês, o maior decréscimo ocorre para os que têm 7 dias e mais e para os menores de um dia, no grupo de 6 a 23 horas. Ou seja, a mortalidade infantil no Estado do Rio de Janeiro decresceu tanto menos quanto mais se aproximou do momento do nascimento.

A comparação com outros países que já reduziram suas taxas de mortalidade infantil a níveis inferiores aos do Brasil pode colaborar na compreensão do problema que se estuda no presente trabalho. Tomou-se como ponto inicial cronológico, desta comparação, o ano de 1960 para os Estados Unidos da América, Reino Unido e Japão. Para estes mesmos países e mais o Chile, levantaram-se informações para os anos de 1978 e 1992, último ano para o qual se dispunha de dados $\left(\mathrm{WHO}^{24}\right)$. O Estado do Rio de Janeiro foi selecionado para compor a Tabela 8 .
Através da distribuição por faixa etária, observase que, em 1960, a mortalidade neonatal nesses países industrializados era muito semelhante a do Estado do Rio de Janeiro, em 1979. No entanto, a mortalidade pós-neonatal era de 2 a 3 vezes maior no Estado, refletindo provavelmente as diferenças existentes nas realidades sociais desses lugares. Com a queda que se processou na mortalidade pós-neonatal, o Rio de Janeiro atingiu em 1993 um coeficiente de mortalidade infantil próximo ao daqueles países em 1960.

$\mathrm{O}$ que de fato mais impressiona não é apenas a diferença desses coeficientes entre os países, mas principalmente as variações observadas na velocidade da queda, para as diversas faixas etárias (Tabela 9). No período 1960-1978 o país, dentre os estudados, que tem a maior redução é o Japão, com uma queda de $7,1 \%$ ao ano, atingindo um dos coeficientes de mortalidade infantil mais baixos do mundo, para a época. Este decréscimo do Japão se fez principalmente às custas dos óbitos que ocorreram na idade de 7 dias e mais de vida, porque, curiosamente, o coeficiente de mortalidade infantil nos menores de um dia já era extremamente baixo. Ao contrário, os Estados Unidos e Reino Unido mostram melhor desempenho entre os menores de 7 dias. No período seguinte, de 1978 a 1992, com a consolidação e difusão da tecnologia perinatal, verifica-se grande impacto na mortalidade neonatal precoce em todos os três países.

Tabela 8 - Coeficientes de mortalidade infantil (óbitos \%o nv) segundo faixa etária em países e anos selecionados e no Estado do Rio de Janeiro.

\begin{tabular}{|c|c|c|c|c|c|c|c|c|c|c|c|c|c|}
\hline \multirow[t]{2}{*}{ Dias de Vida } & \multicolumn{3}{|c|}{$\begin{array}{l}\text { Estados Unidos } \\
\text { da América }\end{array}$} & \multicolumn{3}{|c|}{ Reino Unido } & \multicolumn{3}{|c|}{ Japão } & \multicolumn{2}{|c|}{ Chile } & \multicolumn{2}{|c|}{$\begin{array}{c}\text { Estado do Rio de } \\
\text { Janeiro }\end{array}$} \\
\hline & 1960 & 1978 & 1992 & 1960 & 1978 & 1992 & 1960 & 1978 & 1992 & 1978 & 1989 & 1979 & 1993 \\
\hline$<1$ dia & 10,3 & 5,1 & 3,5 & 7,6 & 3,7 & 1,9 & 2,7 & 1,9 & 1,0 & 7,2 & 4,1 & 7,5 & 7,1 \\
\hline 1-6 dias & 6,4 & 2,9 & 1,3 & 5,7 & 3,4 & 1,4 & 7,9 & 2,3 & 0,8 & 6,6 & 3,1 & 8,6 & 7,0 \\
\hline $7-27$ dias & 2,0 & 1,5 & 1,0 & 2,2 & 1,6 & 1,0 & 6,4 & 1,0 & 0,6 & 4,7 & 1,9 & 6,7 & 3,3 \\
\hline 28-364 dias & 7,3 & 4,3 & 3,4 & 6,3 & 4,5 & 2,3 & 13,7 & 2,7 & 2,1 & 20,2 & 8,0 & 28,0 & 11,6 \\
\hline$<1$ ano & 26,0 & 13,8 & 9,2 & 21,8 & 13,2 & 6,6 & 30,7 & 7,9 & 4,5 & 38,7 & 17,1 & 50,8 & 29,0 \\
\hline
\end{tabular}

Fontes: World Health Statistics ${ }^{24}$

Tabela 9 - Taxa (\%) de variação anual segundo faixa etária em países e períodos selecionados e no Estado do Rio de Janeiro.

\begin{tabular}{|c|c|c|c|c|c|c|c|c|}
\hline \multirow[t]{2}{*}{ Dias de Vida } & \multicolumn{2}{|c|}{$\begin{array}{c}\text { Estados Unidos } \\
\text { da América }\end{array}$} & \multicolumn{2}{|c|}{ Reino Unido } & \multicolumn{2}{|c|}{ Japão } & Chile & \multirow{2}{*}{$\begin{array}{c}\text { Estado do Rio de } \\
\text { Janeiro } \\
79-93 \\
\end{array}$} \\
\hline & $60-78$ & $78-92$ & $60-78$ & $78-92$ & $60-78$ & $78-92$ & 78-89 & \\
\hline$<1$ dia & $-3,9$ & $-2,7$ & $-4,0$ & $-4,8$ & $-1,9$ & $-4,9$ & $-5,1$ & -0.4 \\
\hline 1-6 dias & $-4,4$ & $-5,7$ & $-2,9$ & $-6,3$ & $-6,5$ & $-8,1$ & $-6,9$ & $-2,8$ \\
\hline $7-27$ dias & $-1,6$ & $-2,9$ & $-1,8$ & $-3,4$ & $-9,8$ & $-3,9$ & $-8,2$ & $-5,3$ \\
\hline 28-364 dias & $-2,9$ & $-1,7$ & $-1,9$ & $-4,8$ & $-8,6$ & $-1,9$ & $-8,4$ & $-7,3$ \\
\hline$<1$ ano & $-3,5$ & $-2,9$ & $-2,8$ & $-5,0$ & $-7,1$ & $-4,3$ & $-7,4$ & $-4,7$ \\
\hline
\end{tabular}

Fontes: World Health Statistics ${ }^{24}$ 
O Chile, na década de 80 , apesar de conhecida como a década perdida para os países do terceiro mundo, mostrou uma queda da sua taxa de mortalidade infantil de $7,4 \%$ ao ano, distanciando-se bastante do Estado do Rio que em 15 anos decresceu de apenas $4,7 \%$, sendo o desempenho para o Chile melhor até mesmo para o componente pós-neonatal.

\section{DISCUSSÃO}

A análise temporal da mortalidade infantil no Estado do Rio de Janeiro mostra um grande descenso deste indicador de saúde nos últimos 15 anos. A queda foi maior no Cinturão Metropolitano do que na Capital e Interior do Estado. Esse comportamento, aparentemente paradoxal, explica-se exatamente pelo grande impacto que causou a queda da mortalidade pós-neonatal no coeficiente como um todo. A mortalidade pós-neonatal, determinada principalmente pela ocorrência de óbitos por doenças infecciosas e pela desnutrição, sofreu no período estudado o impacto da ampliação do sistema público de abastecimento de água e da cobertura dos serviços de saúde. O Município do Rio de Janeiro, ao início desta série histórica analisada, já contava com essas benfeitorias acessíveis à maioria da sua população.

De fato, informações dos Censos Demográficos confirmam que, em 1970, o Município do Rio já tinha $83 \%$ dos domicílios ligados à rede pública, enquanto no Cinturão Metropolitano e Interior, apenas 46 e $43 \%$, respectivamente. Em 1980, a situação melhora moderadamente nas 3 áreas, mantendo-se as desigualdades entre os estratos analisados. Mas na década seguinte, o Município do Rio atinge quase a universalidade da cobertura, com $98 \%$ dos domicílios servidos, enquanto o Cinturão Metropolitano e o Interior alcançam as cifras de 73 e $69 \%$.

Os dados censitários também registram uma melhoria no nível de instrução das mulheres. Se em 1970 detectava-se um percentual de $16 \%$ de analfabetas na Capital, em 1991 reduz-se para 7\%. No Cinturão Metropolitano e Interior os valores iniciais eram de 31 e $39 \%$ e, em 1991, de 12 e $15 \%$, respectivamente.

Esses achados são coerentes com o decréscimo da mortalidade pós-neonatal verificada e com as diferenças entre as subáreas. Esrey e Habicht ${ }^{6}$ fazem uma revisão sobre as evidências epidemiológicas dos benefícios para a saúde decorrentes das melhorias sanitárias nos países em desenvolvimento. Chama a atenção, porém, a tendência de convergência dos valores da mortalidade infantil no Município da Capital e Cinturão Metropolitano para o final do perío- do, tanto para o componente pós-neonatal como para o neonatal. Isto induz a pensar na possibilidade de esgotamento do efeito de algumas intervenções ambientais e de alguns benefícios sociais alcançados previamente pela população brasileira em particular das Capitais ${ }^{13,20}$. Além de advertir para a necessidade da adoção de medidas mais específicas que possam dar conta da nova configuração que tem a mortalidade infantil no Estado.

Também não se pode ignorar a colaboração inequívoca que os serviços de saúde deram para o descenso da mortalidade infantil. Houve uma implementação de programas de promoção de saúde e prevenção de doenças, no nível da atenção primária e ambulatorial e nos serviços de saúde pública que, como foi mostrado por Najar e col. ${ }^{15}$, receberam, neste período, grande estímulo governamental, por serem investimentos de baixo custo. A sua atuação fez-se sobretudo através do aumento da cobertura vacinal, do estímulo ao aleitamento materno, do acesso ao sal reidratante oral, da assistência ao pré-natal e ao parto e do controle da natalidade, dentre outros. Por exemplo, o programa de imunização, iniciado com as campanhas, teve, provavelmente, uma importância muito grande para que a população de baixa renda passasse a ter o hábito de consumo de serviços de saúde já que envolvendo a freqüência às Unidades Sanitárias, incluía, conseqüentemente, outros programas de atenção às crianças.

Exibindo um comportamento evolutivo diferente da mortalidade infantil tardia, a mortalidade neonatal decresceu pouco, menos ainda quando se exclui dela o componente relativo às doenças infecciosas e à desnutrição. Em particular, o que ocorreu com a mortalidade no primeiro dia de vida. Com exceção do Município do Rio, não houve redução significativa nessas taxas, encontrando-se, inclusive, aumento no Interior. A situação é ainda mais dramática quando se contempla os óbitos ocorridos na primeira hora após o nascimento, pois, neste caso específico, ocorreu aumento das taxas de mortalidade em todas as três áreas, denunciando uma piora na qualidade da atenção ao parto.

Na tentativa de buscar entender o porquê dos aumentos encontrados na mortalidade, na primeira hora de vida, examinaram-se algumas informações da publicação Assistência Médica, Sanitária, do IBGE. Observou-se que houve diminuição da oferta de leitos hospitalares para a população do Estado do Rio de Janeiro, no período de 1977 a $1992^{7,9}$ e que são grandes as diferenças de cobertura entre as três subregiões do Estado. Na Capital, com predomínio dos leitos do serviço público, as taxas variaram de 7,8 
leitos por 1.000 habitantes, em 1977, para 6,1 em 1992. No Cinturão Metropolitano, de 5,0 leitos para 3,8/1.000 habitantes e no Interior, permaneceram estáveis, em torno de 5,5 leitos/1.000 habitantes, sendo que os leitos nas duas últimas regiões são majoritariamente conveniados com a iniciativa privada.

Porém, ao analisar-se a proporção de óbitos hospitalares ocorridos na primeira hora de vida, ao contrário do esperado, observou-se constância nas proporções, de aproximadamente $97 \%$ para a Capital e de $95 \%$ para as outras regiões, que se mantêm no mesmo patamar durante todo o período de análise. Ou seja, a queda na oferta geral de leitos hospitalares aparentemente não provocou alterações nas coberturas de partos hospitalares. Entretanto, não se pode deixar de considerar a possibilidade de explicação da queda da qualidade na atenção ao parto ser, em parte, devida à diminuição da oferta de leitos obstétricos, o que contribuiria, pelo menos, para o retardo da hospitalização da parturiente, como discutido por Gomes ${ }^{11}$.

Um estudo caso-controle, realizado por Carva$\mathrm{lho}^{4}$, em uma amostra representativa das maternidades da Região Metropolitana do Rio de Janeiro em 1986, retrata a dramática situação do atendimento ao parto e ao recém-nascido. Encontrou-se que 50\% dos prontuários não tinham registro sobre a história gestacional, o pré-natal e o parto; que $21 \%$ deles não tinham registro sobre anamnese e exame físico e mais grave ainda, $40 \%$ não registravam a ausculta aos batimentos cardíacos fetais durante o trabalho de parto. Além disto, um elevado percentual de óbitos foi considerado redutível pelo adequado controle da gravidez e do parto.

O confronto com a experiência dos países industrializados leva à constatação inequívoca de que a mortalidade neonatal do Estado do Rio de Janeiro é extremamente elevada e renitente a uma mudança no tempo. Embora os achados apontem para o benefício da oportunidade de hospitalização para o parto, denunciam, por outro lado, a incapacidade dos nossos serviços de saúde de incorporarem tecnologias simples e eficazes já desenvolvidas. Da comparação com os outros países, inclusive com o Chile, depreende-se que intervenções setoriais específicas seriam capazes de determinar profundas mudanças na tendência da mortalidade infantil no Estado.

É fundamental reiterar a idéia de que os serviços de saúde têm enorme influência na redução da mortalidade infantil. Mesmo o "Black Report", um dos trabalhos mais críticos feitos no Reino Unido sobre as iniqüidades em saúde naquele país, traz como uma das suas recomendações a necessidade de aumentar os investimentos no Serviço Nacional de Saúde, por considerá-lo uma parte importante na estratégia de romper a conexão entre classe social ou pobreza e saúde, particularmente em relação às crianças de pequena idade.

Em contraste ao que ocorre aqui, nos países industrializados o monitoramento desenvolvido para a mortalidade infantil recai primordialmente sobre a primeira semana de vida, e em alguns deles, tem-se verificado uma estagnação da mortalidade pósneonatal e um decréscimo menor da mortalidade neonatal tardia do que da precoce ${ }^{2,3}$. As nossas estatísticas mostram tendência senão de aclive, pelo menos de estabilidade, justamente no período neonatal. Se o grande problema atual enfrentado pela saúde pública, naqueles países, é controlar o nascimento prétermo, que redunda na sobrevivência de bebês extremamente prematuros, com consequiências importantes para o desenvolvimento deles ${ }^{10,12,16}$, os presentes dados revelam problemas opostos, ligados à atenção médico hospitalar, em torno do momento do parto.

Mais grave ainda são as constatações feitas por outros autores, de que os bebês ao nascerem não carecem somente dos cuidados especiais desenvolvidos pela tecnologia médica e já amplamente utilizados em outros países. Muitos dos que não conseguem sobreviver não são prematuros, não têm baixo peso ao nascer, nem demandam cuidados perinatais intensivos, apenas nasceram sem um mínimo de atenção médica, muito embora dentro dos nossos serviços de saúde $e^{4,11}$.

Concluindo, pode-se evidenciar que não está havendo uma sintonia entre a mudança do perfil da mortalidade infantil e a prática dos serviços de saúde no Estado do Rio de Janeiro. Em parte, porque para fazer frente a esta nova realidade há que se definir novas estratégias de intervenção e de monitoramento da realidade sanitária, o que não vem se constituindo em uma prática rotineira dos serviços. Em segundo lugar, para que se consolide um programa de controle de um problema de saúde é necessário que este se constitua em prioridade, com alocação de recursos e de vontade política.

Por último, a ênfase que se dá no presente trabalho ao papel dos serviços de saúde na redução da mortalidade infantil não implica o desconhecimento das determinações sociais e econômicas sobre este indicador, ao contrário, defende-se que é possível atenuálas através da melhoria da qualidade e da democratização da atenção à saúde, responsabilidades sociais específicas e inalienáveis dos serviços de saúde. 


\section{REFERÊNCIAS BIBLIOGRÁFICAS}

1. BLACK, D. et al. Inequalities in health: the black report. London. Penguin Books, 1990.

2. BOUVIER-COLLE, M. H. et al. Causes et facteurs de risque de la mortalite postneonatale en Franceen 1987, d'après une enquete nationale. Rev. Epidemiol. Santè Publique, 39: 43545, 1991.

3. CAMPBELL, M. K. Age at neonatal death in Ontario, 19791987: implications for the interpretation of mortality markers. Paediatr. Perinat. Epidemiol., 7: 426-33, 1993.

4. CARVALHO, M. L. Mortalidade neonatal e aspectos da qualidade da atenção à saúde na Região Metropolitana do Rio de Janeiro em 1986-1987. Rio de Janeiro,1993. [Dissertação de Mestrado-Escola Nacional de Saúde Pública - FIOCRUZ].

5. DUCHIADE, M. P. Mortalidade infantil por pneumonias na Região Metropolitana do Rio de Janeiro em 1986-1987. Rio de Janeiro, 1991. [Dissertação de Mestrado-Escola Nacional de Saúde Pública - FIOCRUZ].

6. ESREY, S. A. \& HABICHT, J. Epidemiologic evidence for health benefits from improved water and sanitation in developing countries. Epidemiol. Rev., 8: 117-28, 1986.

7. FUNDAÇÃO IBGE. Assistência médico-sanitária: base de dados em formato tabular, 1992. Rio de Janeiro, 1995 [formato disquete].

8. FUNDAÇÃO IBGE. Estatísticas do Registro Civil, v. 6-20, 1979-1993. Rio de Janeiro, 1981-1995.*

9. FUNDAÇÃO IBGE. Estatísticas de saúde: assistência médicosanitária, v. 2-14, 1977-1989. Rio de Janeiro, 1978-1994.*

10. FOGER, M. Trends in perinatal, neonatal and post-neonatal mortality in Austria and Tyrol, with special reference to 1979-1988. Pediatr. Padol., 26: 257-62, 1991.

11. GOMES, M. A. S. M. Aspectos da qualidade do atendimento à gestação e ao parto através da percepção das usuárias. Rio de Janeiro, 1995. [Dissertação de Mestrado - Instituto Fernandes Figueira - FIOCRUZ].

12. MC CORMICK, M. C. \& WISE, P. H. Infant mortality. Curr. Opin. Pediatr., 5: 552-7, 1993.

13. MONTEIRO, C. A. Contribuição para o estudo do significado da evolução do coeficiente de mortalidade infantil no Município de São Paulo, S. P. (Brasil), nas três últimas décadas (1950-1979). Rev. Saúde Pública, 16: 7-18, 1982.
14. MONTEIRO, M. F. G. Considerações sobre os fatores socio- econômicos e diferenciais de mortalidade infantil nas Regiões Metropolitanas do Brasil, calculados através do método caso-controle. In: Fundação IBGE. Perfil estatístico de crianças e mães do Brasil - aspectos socioeconômicos da mortalidade infantil em áreas urbanas. Rio de Janeiro, 1986.

15. NAJAR, A. L. et al. Relatório final do projeto de investigação "Relação saúde e qualidade ambiental: uma abordagem territorializada para a região metropolitana do Rio de Janeiro". Rio de Janeiro, Escola Nacional de Saúde Pública,1995.

16. NISHIDA, H. Outcome of infant born preterm, with special emphasis on extremely low birthweight infants. Baillieres. Clin. Obstet. Gynaecol., 7: 611-31, 1993.

17. OLIVEIRA, L. A. P. A mortalidade infantil recente e a dinâmica social. In: Simões C. C. S. org. Perfil estatístico de crianças e mães do Brasil - mortalidade infantil e saúde na década de 80. Rio de Janeiro, FIBGE, 1989.

18. RUTSTEIN, D. D. et al. Measuring the quality of medical care. A clinical method. N. Engl. J. Med., 294: 582-8, 1976.

19. SABROZA, P. C. et al. Saúde, ambiente e desenvolvimento: alguns conceitos fundamentais. In: Leal, M. C. et al. org. Saúde, ambiente e desenvolvimento. São Paulo, Hucitec/ Abrasco, 1992. v. 1, p. 45-93.

20. SIMÕES, C. C. S. \& ORTIZ, L. P. A mortalidade infantil nos anos 80: textos para discussão. Rio de Janeiro, FIBGE, 1988.

21. SZWARCWALD, C. L. et al. Mortalidade infantil: o custo social do desenvolvimento brasileiro. In: Leal, M. C. et al. org. Saúde, ambiente e desenvolvimento. São Paulo, Hucitec/ Abrasco, 1992. v. 2, p. 251-78.

22. SZWARCWALD, C. L. et al. Tendências da mortalidade infantil no Brasil nos anos 80. Inf. Epidemiol. SUS, 1: 32-5, 1992.

23. VICTORA, C. G. et al. Epidemiologia da desigualdade. São Paulo, Hucitec, 1989.

24. WORLD HEALTH ORGANIZATION. World Health Statistics, 1960-1964; 1978-1981; 1990-1993. Geneva, 1963-1968; 1978-1981; 1991-1994*. 